\title{
Coping with CDMA Asynchronicity in Compressive Sensing Multi-User Detection
}

\author{
Henning F. Schepker, Carsten Bockelmann and Armin Dekorsy \\ Department of Communications Engineering \\ University of Bremen, Bremen, Germany \\ email: \{schepker, bockelmann, dekorsy\}@ant.uni-bremen.de
}

\begin{abstract}
The growing field of Machine-to-Machine communication requires new physical layer concepts to meet future requirements. In previous works it has been shown for a synchronous CDMA transmission that Compressive Sensing (CS) detectors are capable of jointly detecting both activity and data in multi-user detection (MUD). However, many practical applications show some degree of asynchronicity. In order to reduce transmitter complexity, we propose an enhanced CS MUD that detects the delay in addition to activity and data. This solves synchronicity issues for scenarios with a known maximum delay, without requiring signaling or pre-compensation of asynchronicity.
\end{abstract}

\section{INTRODUCTION}

The field of wireless Machine-to-Machine communication is expected to grow tremendously in the future. This calls for new and adapted physical layer concepts, as system requirements differ from common applications such as high data rate access. For uplink transmission in a sensor network using CDMA, it has already been shown in [1]-[3] that reliable joint detection of both activity and data is possible using Compressive Sensing (CS) in Multi-User Detection (MUD) [4], [5], even for overloaded CDMA systems. The main requirement for CS MUD is that the transmission is sporadic, i.e., each transmitter is inactive most of the time.

The previous investigations of CS MUD in the context of CDMA were focused on synchronous CDMA transmission. However, synchronicity first has to be achieved by appropriate techniques or protocols. While such techniques are known, e.g., estimating asynchronicity after the transmission of fixed synchronization words, or pre-compensation of asynchronicity at the transmitter based on feedback, the transmission from sensor nodes to the aggregation node can be made much simpler if this is not required [6]. As sensor nodes ideally should have low complexity, we propose an asynchronous transmission scheme where delays are estimated as part of the CS MUD.

The principle approach of detecting delays in CS MUD is shown for a random on/off access channel (RACH) in [7]. We expand this idea and propose a modified CS detection that simultaneously detects activity, data and delay for all nodes. For this detection we propose a modified greedy algorithm that makes use of the knowledge that the transmission from a node can only be received at one specific delay. We also provide numerical results showing how reliable CS detection is for a given amount of asynchronicity.

\section{SySTEM MODEL}

\section{A. CDMA Uplink Transmission}

We consider a CDMA uplink transmission, where $K$ sensor nodes communicate with a central aggregation node. Here, we assume that the transmissions from the sensor nodes are sporadic, i.e., the sensor nodes are only active on occasion. As a model for sensor node activity, we assume that each sensor node is active for a short period of time with a given activity probability $p_{a}$. Further, we assume that this activity probability is identical for all sensor nodes and rather small, i.e., $p_{a} \ll 1$. For a large number of nodes $K$ this is a valid assumption for practical applications.

For the transmitter setup of the sensor nodes we assume that an active node $k_{\mathrm{a}}$ transmits a data frame of $N_{\mathrm{F}}$ modulated symbols $\mathbf{d}_{k_{\mathrm{a}}} \in \mathcal{A}^{N_{\mathrm{F}}}$, where $\mathcal{A}$ is the modulation alphabet. The dimension of $N_{\mathrm{F}}$ is predetermined by the higher layers. For simplicity, we assume that BPSK is used for modulation. This restriction simplifies the notation without loss of generality, as we consider a real-valued model. Other modulation schemes can easily be applied. An inactive node $k_{\mathrm{i}}$ does not transmit any data, thus we model the transmitted symbols as zero symbols, i.e., $\mathbf{d}_{k_{\mathrm{i}}} \in\{0\}^{N_{\mathrm{F}}}$. Therefore, each sensor transmits frames of $N_{\mathrm{F}}$ consecutive symbols drawn from the so-called augmented alphabet $\mathcal{A}_{0}=\{\mathcal{A} \cup 0\}$, which is the BPSK alphabet $\mathcal{A}$ augmented and extended by the zero symbol to indicate inactivity.

For simplicity, we assume the uplink transmission is facilitated with Pseudo Noise $(\mathrm{PN})$ sequences $\mathbf{s}_{k}$, where the spreading factor $N_{\mathrm{S}}$, i.e., the number of chips per information symbol, is identical for all sensor nodes. Further, we assume that $\mathrm{PN}$ sequences $\mathbf{s}_{k}$ are constant for a frame.

As a channel model, we assume that transmitted symbols are distorted by a node specific frequency selective channel $\mathbf{h}_{k} \in \mathbb{R}^{L_{\mathrm{h}}}$ of length $L_{\mathrm{h}}$ chips, which is constant for a frame, i.e., a block-fading channel. Here, we assume that the data frames from all nodes are received with no interference by either control signals or other data frames, such that we only have to model interferences within a multi-user data frame.

\section{B. Asynchronous CDMA Chip-rate Model}

Assuming synchronicity in the chip-rate model, the $m=N_{\mathrm{F}} N_{\mathrm{S}}+L_{\mathrm{h}}-1$ received chips from node $k$ are defined 
by $\mathbf{R}_{k} \mathbf{d}_{k}$, where $\mathbf{R}_{k} \in \mathbb{R}^{m \times N_{\mathrm{F}}}$ is given as [6]

$$
\mathbf{R}_{k}=\left(\begin{array}{ccc}
r_{k, 0} & 0 & 0 \\
\vdots & 0 & 0 \\
r_{k, N_{\mathrm{S}}+1} & r_{k, 0} & 0 \\
\vdots & \vdots & 0 \\
0 & \vdots & \ddots
\end{array}\right) .
$$

The vector $\mathbf{r}_{k} \in \mathbb{R}^{\left(N_{\mathrm{S}}+L_{\mathrm{h}}-1\right)}$ is determined by the convolution of the spreading sequence $\mathbf{s}_{k}$ with the channel impulse response $\mathbf{h}_{k}$, assuming perfect channel state information. As both the channel $\mathbf{h}_{k}$ and the PN sequence $\mathbf{s}_{k}$ are constant for a frame $\mathbf{d}_{k}$, the non-zero column entries of $\mathbf{R}_{k}$ are identical, but shifted to different rows.

In contrast to the synchronicity assumption, many practical applications show some degree of asynchronicity between nodes. In order to keep the complexity of the transmitters small, we incorporate this asynchronicity in the MUD model and detect the delays simultaneously with the activity and data. To address asynchronicity, we assume that the individual nodes have an unknown delay of $\tau_{k}$ chips and that the delay of each node is independently uniformly distributed in the range of $0 \leq \tau_{k} \leq \tau_{\max } \forall k$. One example how this can be achieved is the transmission of a frame clock signal, such that the sensor nodes transmit after receiving this frame clock signal. As the maximum delay $\tau_{\max }$ can only be estimated, we denote the estimate used to build the detection model at the receiver as $\tau_{\text {model }}$. In the following, we consider all delays to be measured in chip-rate.

In order to include asynchronicity in the detection, we introduce a delay hypothesis for each possible delay $\hat{\tau}_{k_{\mathrm{V}}}$ of node $k$, represented by a virtual node $k_{\mathrm{V}}$. Each virtual node $k_{\mathrm{V}}$ of node $k$ models the reception of frame $\mathbf{d}_{k_{\mathrm{V}}}$ spread with $\mathbf{R}_{k}$ at a delay of $\hat{\tau}_{k_{\mathrm{v}}}$. We write the set of all virtual nodes of node $k$ as $\mathcal{V}_{k}=\left\{k_{\mathrm{V}} \mid\left\lceil k_{\mathrm{V}} /\left(\tau_{\text {model }}+1\right)\right\rceil=k\right\}$. As delay hypotheses have to be considered for all $K$ nodes, a total of $K_{\mathrm{V}}=K\left(\tau_{\text {model }}+1\right)$ virtual nodes are introduced. The multiuser vector $\mathbf{x}$ for this model does not only contain $N_{\mathrm{F}}$ symbols for each node $k$, but rather for each virtual node $k_{\mathrm{V}}$. Thus, $\mathbf{x} \in \mathbb{R}^{\left(K_{\mathrm{V}} N_{\mathrm{F}}\right)}$ is given as

$$
\mathbf{x}=\left[\mathbf{d}_{1}^{T}, \ldots, \mathbf{d}_{K_{\mathrm{V}}}^{T}\right]^{T} .
$$

Consequently, for this multi-user vector the chip-rate MUD problem is given as

$$
\mathbf{y}=\mathbf{A x}+\mathbf{n}
$$

where $\mathbf{n} \in \mathbb{R}^{\left(m+\tau_{\text {model }}\right)}$ is real-valued AWGN noise $\mathcal{N}\left(0, \sigma_{n}^{2}\right)$ and the system matrix $\mathbf{A} \in \mathbb{R}^{\left(m+\tau_{\text {model }}\right) \times\left(K_{\mathrm{V}} N_{\mathrm{F}}\right)}$ is given by

$$
\mathbf{A}=\left(\begin{array}{cccccc}
\mathbf{R}_{1} & 0 & 0 & \mathbf{R}_{K} & 0 & 0 \\
\vdots & \mathbf{R}_{1} & 0 & \vdots & \mathbf{R}_{K} & 0 \\
0 & \vdots & \ddots & 0 & \vdots & \ddots
\end{array}\right)
$$

Due to the delay hypotheses, the dimension of the detection problem increases with increasing $\tau_{\text {model }}$. More specifically, the dimension of the multi-user vector increases by a factor of $\left(\tau_{\text {model }}+1\right)$, while the measurements are increased by $\tau_{\text {model }}$. This means that the detection problem becomes stronger under-determined with increasing $\tau_{\text {model }}$. However, increasing $\tau_{\text {model }}$ does not increase the number of non-zero elements in $\mathbf{x}$, as the number of active nodes does not increase. Therefore, even though the problem is stronger under-determined it can still be reliably recovered with CS detectors, as the fraction of non-zeros decreases.

\section{Detection Model}

As the size of the multi-user vector $\mathbf{x}$ is given by $n=K_{\mathrm{V}} N_{\mathrm{F}}$, the computational complexity quickly gets prohibitive for large frame sizes $N_{\mathrm{F}}$, large amount of nodes $K$ or large maximum delays $\tau_{\text {model }}$. In order to be able to implement the detection in practical communication systems, the dimension of the detection problem has to be reduced. Therefore, (3) is divided into $\nu=1, \ldots, N_{\mathrm{F}} / L$ sub-problems, each considering $L$ consecutive transmit symbols per virtual node. Thus, each of those sub-problems has a reduced dimension of $m_{\nu}=L N_{\mathrm{S}}+L_{\mathrm{h}}-1+\tau_{\text {model }}$ and $n_{\nu}=K_{\mathrm{V}} L$. All subproblems are determined by a system matrix $\mathbf{A}_{\nu} \in \mathbb{R}^{m_{\nu} \times n_{\nu}}$, which is the same for all $\nu$, as both PN sequences and channels are assumed to be constant for an entire frame. In order to simplify the model, we neglect the ISI between sub-problems in the detection. The sub-problem dimension $L$ is chosen in the system design, and defines the tradeoff between reduced complexity and detection accuracy.

A common requirement for high detection accuracy in CS literature is that the system matrix has unit norm columns, i.e., columns with identical $\ell_{2}$-norm. However, $\mathbf{A}$ does not have this property as the channel coefficients $\mathbf{h}_{k}$ only have average values that satisfy $\left\|\mathbf{h}_{k}\right\|_{2}^{2}=1$. Thus, due to the random influence of the channel, the system matrix $\mathbf{A}_{\nu}$ does not have unit norm columns in most cases. To solve this problem, we modify the system matrix $\mathbf{A}_{\nu}$ in each of the $\nu=1, \ldots, N_{\mathrm{F}} / L$ $\mathrm{CS}$ detection sub-problems to have unit norm columns, as shown in [8], such that

$$
\mathbf{y}_{\nu}=\tilde{\mathbf{A}}_{\nu} \tilde{\mathbf{x}}_{\nu}+\mathbf{n}_{\nu}
$$

where $\mathbf{n}_{\nu} \in \mathbb{R}^{N_{\mathrm{S}} L}$ is real-valued AWGN noise $\mathcal{N}\left(0, \sigma_{n}^{2}\right)$, $\mathbf{y}_{\nu} \in \mathbb{R}^{N_{\mathrm{S}} L}$ denotes the received sub-vector at the aggregation node, and $\tilde{\mathbf{x}}_{\nu}=\tilde{\mathbf{H}}_{\nu} \mathbf{x}_{\nu}$. Here, $\tilde{\mathbf{H}}_{\nu}$ is a diagonal matrix containing the column $\ell_{2}$-norms of $\mathbf{A}_{\nu}$, and $\tilde{\mathbf{A}}_{\nu}$ is the matrix $\mathbf{A}_{\nu}$ with each column divided by its $\ell_{2}$-norm. The values in $\tilde{\mathbf{x}}_{\nu}$ for a virtual node $k_{\mathrm{V}}$ are scaled according to the column $\ell_{2}$-norm, such that $\tilde{\mathcal{A}}_{0}=\left\{0, \pm\left\|\mathbf{A}_{\nu, k_{\mathrm{V}}}\right\|_{2}\right\}$. For simplicity, we treat the elements as being continuous during the detection and then quantize the estimated frame for each virtual node to $\hat{\mathbf{d}}_{k_{\mathrm{V}}} \in \mathcal{A}_{0}^{N_{\mathrm{F}}}$.

\section{Compressive Sensing Multi-User Detection}

\section{A. Restricted Group Orthogonal Matching Pursuit (rGOMP)}

The theory of Compressive Sensing (CS) is focused on the reconstruction of compressible signals by recovery of sparse signals even from under-determined equation systems [4], [5]. 


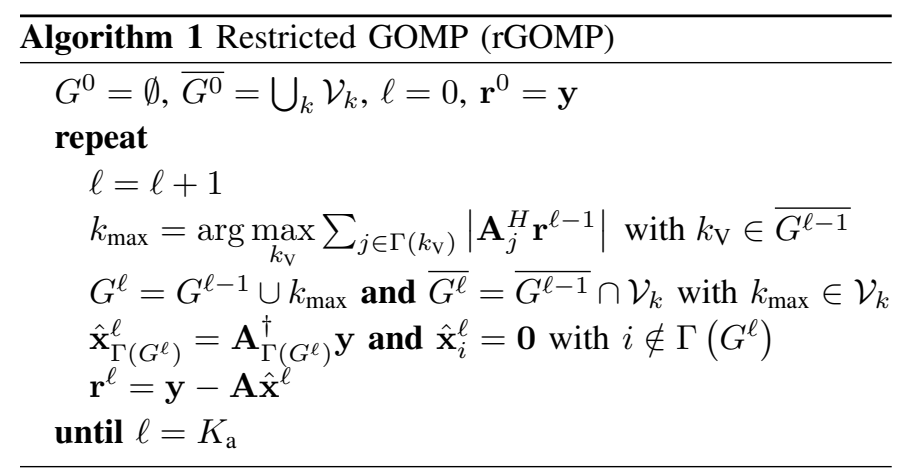

While there are many different approaches for CS, e.g., [9][11], in this paper we focus on CS MUD using known greedy algorithms. These algorithms are in general more efficient, but less accurate than solving convex optimization problems, such as [9].

In our scenario, the node activity is constant for a frame, and thus a group of elements in $\mathrm{x}$ that belong to the same virtual node $k_{\mathrm{V}}$ is either all zero or non zero. This property is called block-sparse or group-sparse. A greedy algorithm that makes use of this property is the Group Orthogonal Matching Pursuit (GOMP) [12] algorithm.

In our system model, in addition to $\mathrm{x}$ being block-sparse, we have additional information about activity of virtual nodes. As a transmitted frame from node $k$ can only arrive at one delay $\tau_{k}$, only one virtual node $k_{\mathrm{V}}$ of node $k$ can be active for a given frame. To exploit this knowledge, we propose a restricted version of the GOMP. This approach is different from [7], where neither group-sparsity nor the additional information limiting the valid activity combinations were exploited.

In order to explain the restricted GOMP, we first introduce our notation: For simplicity, we omit sub-problem index $\nu$ in the notation of the algorithm, even though the detector is applied to (5). $G$ is a set of group-indices and $\bar{G}$ is the set of all valid group-indices not in $G . \Gamma\left(k_{\mathrm{V}}\right)$ specifies the vectorindices corresponding to group-index $k_{\mathrm{V}}$ and $\Gamma(G)$ specifies the vector-indices corresponding to any group in $G . \mathbf{A}_{\Gamma(G)}$ specifies the sub-matrix which only contains those columns with vector-indices in $\Gamma(G)$, and likewise $\mathbf{x}_{\Gamma(G)}$ contains only those elements of $\mathbf{x}$ with vector-indices in $\Gamma(G) . \mathbf{x}^{\ell}, \mathbf{A}^{\ell}$ and $G^{\ell}$ each specify the respective variable during the $\ell^{\text {th }}$ iteration. Herein, $\mathbf{A}^{\dagger}$ is the Moore-Penrose pseudoinverse of $\mathbf{A}$, and $\mathbf{A}^{H}$ the Hermitian matrix of $\mathbf{A}$.

The rGOMP iteratively determines the support of $\hat{x}$, i.e., the location of the non-zero elements. During each iteration, the rGOMP determines the group of columns $k_{\max }$ from the set of valid choices $\bar{G}$ that has the highest correlation to the previous residual $\mathbf{r}^{\ell-1}$. For a generic GOMP, $\bar{G}$ is the complementary set of $G$. Here, using the additional knowledge regarding active virtual nodes, we define that set $\bar{G}$ contains those sets of virtual node indices $\mathcal{V}_{k}$, where no virtual node $k_{\mathrm{V}} \in \mathcal{V}_{k}$ is in $G$, i.e.,

$$
\bar{G}=\bigcup_{\mathcal{V}_{k} \cap G=\emptyset} \mathcal{V}_{k}=\left\{k_{\mathrm{V}}: k_{\mathrm{V}} \in \mathcal{V}_{k} \wedge \mathcal{V}_{k} \cap G=\emptyset\right\}
$$

This ensures that for each node $k$ only one virtual node $k_{\mathrm{V}} \in \mathcal{V}_{k}$ can be estimated as active. Afterwards, the rGOMP calculates the least-square (LS) estimate $\hat{\mathbf{x}}^{\ell}$ based on the current and all previous node choices, and updates the residual $\mathbf{r}^{\ell}$. For simplicity, we assume that the algorithm is terminated after a number of iterations equal to the number of active nodes $K_{\mathrm{a}}$. For implementation, an appropriate termination criterion needs to be found that is well suited for the current scenario.

\section{B. Data Aggregation}

Due to the independent detection of each sub-problem, the delays will in general be estimated differently for each subproblem. If the delays are required for further processing, a joint delay estimation across all sub-problems has to be performed. Here, we assume that only the received data is required by the higher layers, and therefore we can discard the delays. For this, we first have to collect the estimated data and activity for each virtual node $k_{\mathrm{V}}$. This is done by collecting the $L$ estimated symbols $\hat{\mathbf{d}}_{k_{\mathrm{v}}, \nu}$ from each sub-problem $\nu$ into the estimated frame $\hat{\mathbf{d}}_{k_{\mathrm{v}}}$. Afterwards, we sum up the estimated symbols across all virtual nodes $k_{\mathrm{V}} \in \mathcal{V}_{k}$ of node $k$ with

$$
\hat{\mathbf{d}}_{k}=\sum_{k_{\mathrm{v}} \in \mathcal{V}_{k}} \hat{\mathbf{d}}_{k_{\mathrm{v}}} \text {. }
$$

This reduces the node dimension to that of the original $K$ nodes and thus discards the estimated delay information. Due to the restriction of the rGOMP only one delay hypothesis $k_{\mathrm{V}}$ of node $k$ can be estimated as active in each sub-problem, ensuring that in (7) no superposition of different data estimations occurs.

\section{Simulation Results}

In this section, we will discuss simulation results for simultaneously detecting activity, data and delay in an asynchronous CDMA transmission using the rGOMP. For comparison of the symbol error rate (SER) over the augmented alphabet $\mathcal{A}_{0}$, performance results for two detectors are given: On the one hand, the best case performance and thus the lower bound for the rGOMP is given by LS estimation for known activity and delay. We call this ideal detector the oracle $L S$. On the other hand, results of rGOMP detection for a synchronous CDMA transmission are given, i.e., $\tau_{\max }=0$.

As a simulation setup, we focus on an overloaded CDMA system. More specifically, we consider a transmission from $K=128$ sensor nodes, where each node transmits using a PN sequence of length $N_{\mathrm{S}}=32$, which leads to detection equations that are under-determined by a factor of four. For comparison, we will also consider a smaller system with only $K=32$ sensor nodes and spreading factor of $N_{\mathrm{S}}=8$. Unless otherwise noted, we assume that sensor nodes are only active with a probability of $p_{a}=0.02$, so that the number of active nodes is on average much smaller than $K$. Furthermore, the frame length is $N_{\mathrm{F}}=56$ symbols and the channel is modeled by $L_{\mathrm{h}}=6$ i.i.d. Gaussian distributed taps with an exponential decaying power delay profile. The CS detection uses $L=8$ consecutive symbols per sub-problem. For the simulations, we 


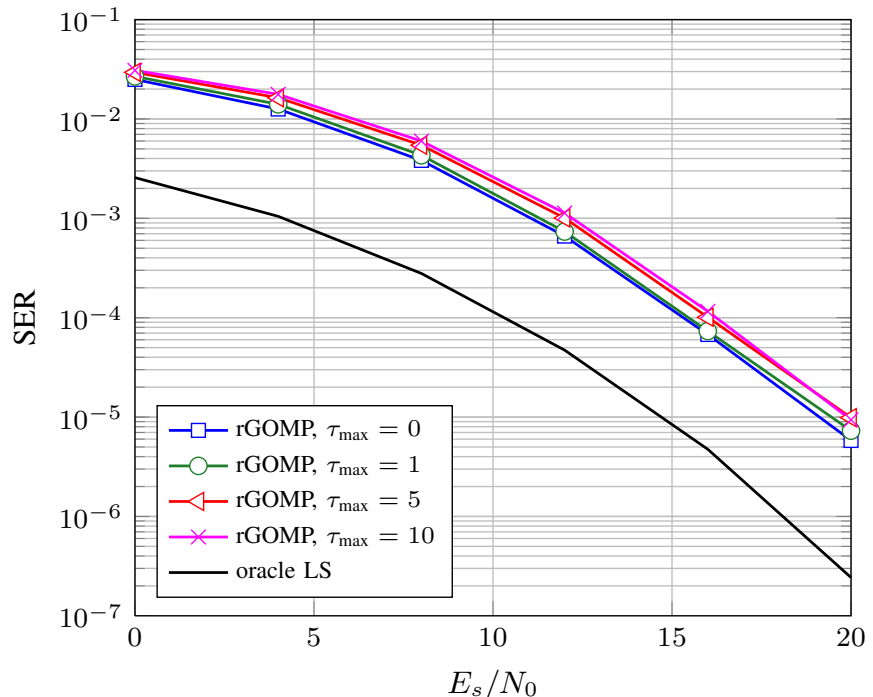

Fig. 1. Symbol Error Rate over the Augmented Alphabet for $K=128$ and $N_{\mathrm{S}}=32$.

initially assume that $\tau_{\text {model }}=\tau_{\max }$. Later, we show results for a mismatch of $\tau_{\text {model }}$ and $\tau_{\text {max }}$.

The SER over the augmented alphabet $\mathcal{A}_{0}$ contains both errors due to incorrect activity detection and errors due to incorrect data detection. Fig. 1 shows the SER of the rGOMP for different maximum delays $\tau_{\max }$. From these results we can see that the SER of the rGOMP increases slightly with increasing $\tau_{\max }$. A small maximum delay $\tau_{\max }=1$ only has a minor influence on the detection performance, while a delay of $\tau_{\max }=5$ approximately causes a $0.8 \mathrm{~dB}$ loss. Once the maximum delay has reached a certain value it no longer increases the SER significantly, as indicated by the performance for $\tau_{\max }=10$. However, this only holds as long as symbol synchronicity is still adhered. This can be seen by the SER results for a smaller system shown in Fig. 2. Here, the delay $\tau_{\max }=10$ is larger than the spreading factor $N_{\mathrm{S}}=8$, and therefore symbol synchronicity is no longer guaranteed. This causes a mismatch of symbols and measurements for some delays, and thus significantly increases the amount of data detection errors. Thus, the transmission should be designed such that $\tau_{\max }<N_{\mathrm{S}}$ to avoid data detection errors due to loss of symbol synchronicity.

When comparing the rGOMP to the oracle LS in Fig. 1, we can see that the errors of the rGOMP are primarily activity errors, as the oracle LS does not result in any activity errors and its SER performance is given by the LS data estimation. There are two types of activity errors: On the one hand estimating an active symbol as inactive, called missed detection, and on the other hand estimating an inactive symbol as active, called false alarm. The resulting Missed Detection Rate (MDR) and False Alarm Rate (FAR) in Fig. 3 indicate that the asynchronicity has a similar influence on the activity errors as on the SER. This is due to the fact that the SER is primarily determined by activity errors.

In addition to the previous SER results, Fig. 4 shows

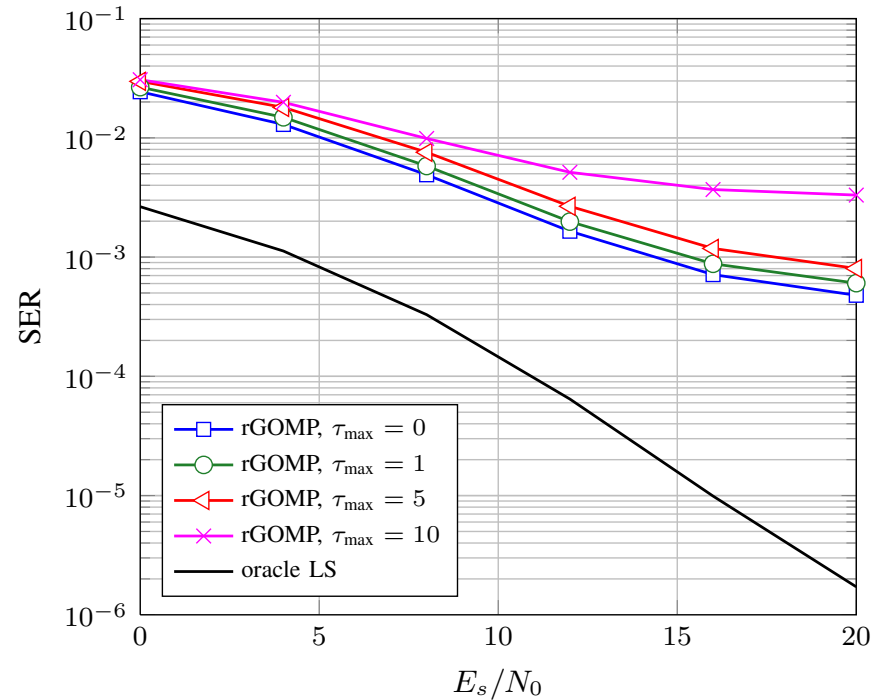

Fig. 2. Symbol Error Rate over the Augmented Alphabet for $K=32$ and $N_{\mathrm{S}}=8$.

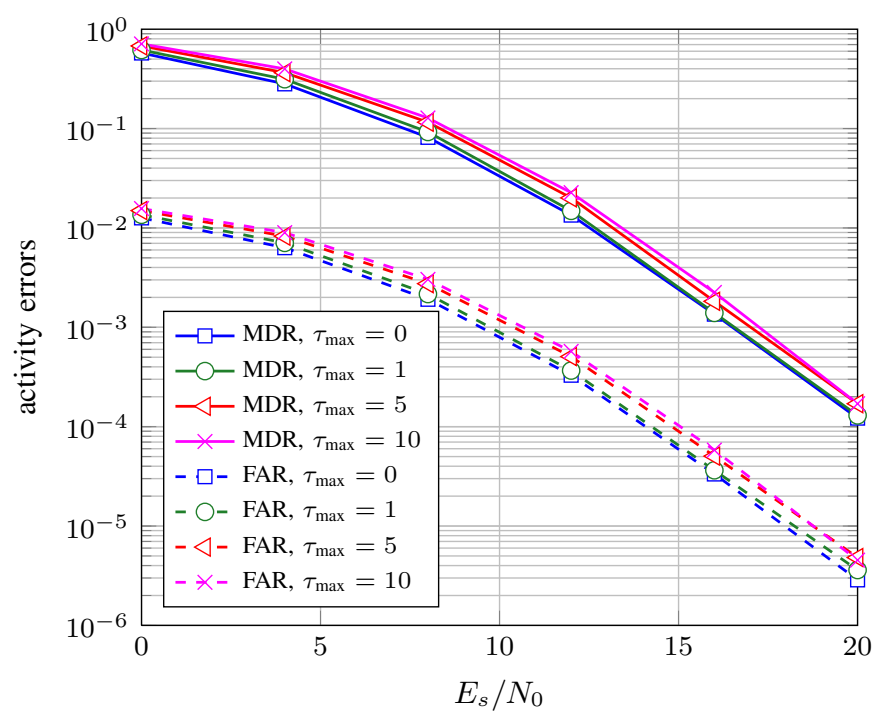

Fig. 3. Activity Errors of rGOMP for different values of $\tau_{\max }, K=128$, and $N_{\mathrm{S}}=32$.

the SER of the rGOMP for fixed $E_{s} / N_{0}=12 \mathrm{~dB}$ and a range of $p_{a}=0.01, \ldots, 0.1$. Here, the main observation is that for the asynchronous case the higher $\tau_{\max }$ is, the worse rGOMP scales with $p_{a}$. This means that for a given $\tau_{\text {model }}$ increasing the activity probability $p_{a}$ also results in a larger performance difference to the synchronous case. In general, a higher activity probability $p_{a}$ makes higher activity levels more likely, and thus increases the average number of active nodes. By introducing the delay hypotheses, $\tau_{\max }$ additional nodes that are correlated to the original node $k$ are added to the detection problem. This makes differentiating between users in the MUD less reliable, which increases the detection errors, especially for higher activity levels and larger $\tau_{\max }$.

Finally, Fig. 5 shows the influence on the SER of a mismatch of the delay $\tau_{\text {model }}$, used to build the detection model at 


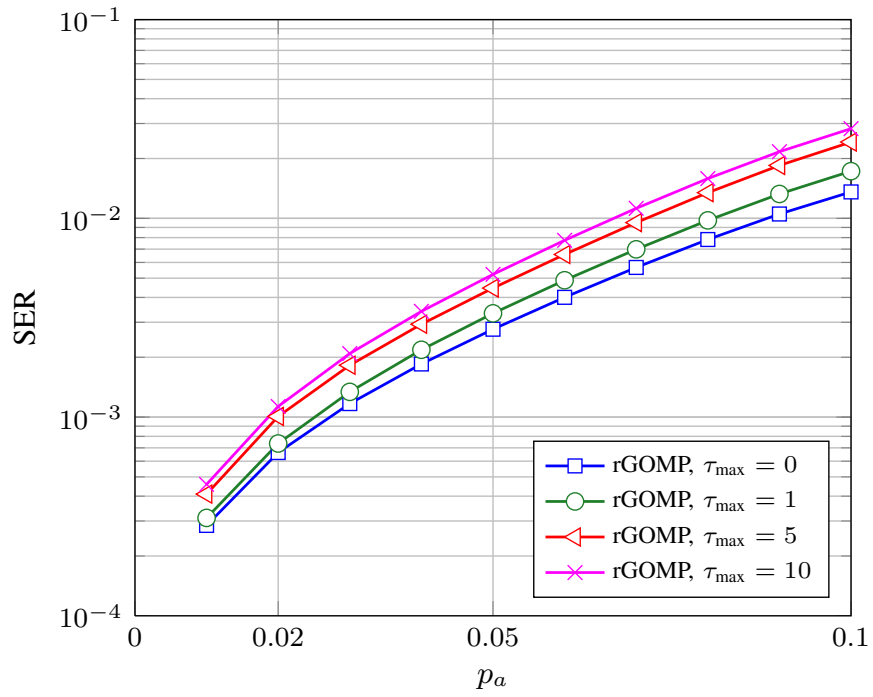

Fig. 4. Symbol Error Rate for a range of activity probabilities $p_{a}=0.01, \ldots, 0.1, E_{s} / N_{0}=12 \mathrm{~dB}, K=128$, and $N_{\mathrm{S}}=32$.

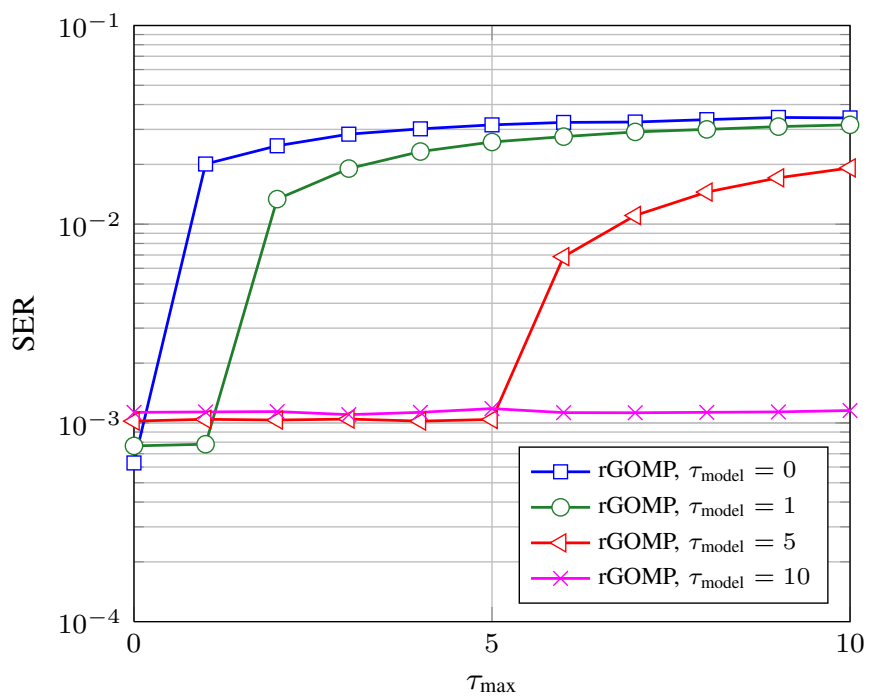

Fig. 5. Symbol Error Rate for different $\tau_{\text {model }}$ over a range of $\tau_{\max }$, for $E_{s} / N_{0}=12 \mathrm{~dB}, K=128$, and $N_{\mathrm{S}}=32$.

the receiver, and the maximum delay $\tau_{\max }$ that actually occurs during the transmission. For cases where $\tau_{\text {model }} \geq \tau_{\max }$, the performance is independent of the actual value of $\tau_{\max }$. This can be seen by the constant SER for each $\tau_{\text {model }}$ as long as $\tau_{\text {model }} \geq \tau_{\max }$. This indicates that the SER performance is mainly determined by the number of delay hypotheses introduced and not the actual delays. Also as previously observed, lower $\tau_{\text {model }}$ result in more accurate detection. In the opposite case, where $\tau_{\text {model }}<\tau_{\max }$, the SER increases significantly for increasing $\tau_{\max }$. This is due to the fact that with increasing $\tau_{\max }$, there is an increasing probability that delays occur that are larger than those expected by the receiver. As those delays are not correctly captured in the detection, and transmitted values at those delays are perceived as additional interference, which increases the error probability. When comparing the SER for different value of $\tau_{\text {model }}$, we see that for $\tau_{\text {model }} \geq \tau_{\max }$ smaller $\tau_{\text {model }}$ result in more detection errors. From the results of both cases it is clear that larger $\tau_{\text {model }}$ result in a detection that is less influenced by a mismatch of $\tau_{\text {model }}$ and $\tau_{\max }$. This comes at the cost of both a slightly higher SER for small maximum delays $\tau_{\max }$ and a higher computational complexity.

\section{CONClusion}

In this paper, we have investigated how CS MUD can be performed for asynchronous CDMA transmission. To this end, we proposed a detection model that allows for simultaneous detection of activity, data and delays, by expanding the detection with delay hypotheses. Additionally, we introduced a restricted GOMP algorithm that makes use of the knowledge that for each node only one delay hypothesis can be true. We have shown that even delays up to the spreading factor just slightly increase the detection errors, as long as the maximum delay is estimated appropriately. Thus, for scenarios where delays have a known limit, asynchronicity can be fully handled by CS MUD. This idea can be further improved by more sophisticated CS MUD algorithms, such that the SER performance of perfect activity detection in a synchronous system can be approached.

\section{REFERENCES}

[1] H. Zhu and G. B. Giannakis, "Exploiting sparse user activity in multiuser detection," IEEE Transactions on Communications, vol. 59, no. 2, pp. 454-465, February 2011.

[2] H. F. Schepker and A. Dekorsy, "Sparse multi-user detection for CDMA transmission using greedy algorithms," in 8th International Symposium on Wireless Communication Systems, Aachen, Germany, November 2011.

[3] H. Schepker and A. Dekorsy, "Compressive sensing multi-user detection with block-wise orthogonal least squares," in IEEE 75th Vehicular Technology Conference, Yokohama, Japan, May 2012.

[4] D. L. Donoho, "Compressed sensing," IEEE Transactions on Information Theory, vol. 52, no. 4, pp. 1289-1306, April 2006.

[5] E. J. Candès, J. Romberg, and T. Tao, "Robust uncertainty principles: Exact signal reconstruction from highly incomplete frequency information," IEEE Transactions on Information Theory, vol. 52, no. 2, pp. 489-509, February 2006.

[6] S. Verdú, Multiuser Detection. Cambridge, U.K.: Cambridge Univ. Press, November 1998.

[7] L. Applebaum, W. Bajwa, M. F. Duarte, and R. Calderbank, "Asynchronous code-division random access using convex optimization," Physical Communication, vol. 5, no. 2, pp. 129 -147, 2012.

[8] S. Rangan, A. Fletcher, and V. Goyal, "Asymptotic analysis of MAP estimation via the replica method and applications to compressed sensing," IEEE Transactions on Information Theory, vol. 58, no. 3, pp. 1902 -1923, March 2012.

[9] R. Tibshirani, "Regression shrinkage and selection via the lasso," Journal of the Royal Statistical Society, Series B, vol. 58, no. 1, pp. 267-288, 1996.

[10] Y. Pati, R. Rezaiifar, and P. Krishnaprasad, "Orthogonal matching pursuit: Recursive function approximation with applications to wavelet decomposition," Signals, Systems and Computers, vol. 1, pp. 40-44, November 1993.

[11] T. Blumensath and M. E. Davies, "Iterative hard thresholding for compressed sensing," Applied and Computational Harmonic Analysis, vol. 27 , no. 3 , pp. $265-274,2009$.

[12] A. Majumdar and R. K. Ward, "Fast group sparse classification," Electrical and Computer Engineering, Canadian Journal of, vol. 34, no. 4, 2009. 